\title{
Massive Splenic Infarction Secondary to Distal Pancreatectomy for a Blunt Abdominal Trauma
}

\author{
Iosvany Rivero Hernández ${ }^{1}$, Antonio Calvo Durán ${ }^{1}$, Ana Diez Núñez ${ }^{1}$, Juan Alija Castro ${ }^{2}$, \\ Vicente Vega Ruiz ${ }^{1}$
}

${ }^{1}$ Department of General and Digestive Surgery, Universitary Hospital of Puerto Real, Puerto Real, Spain

${ }^{2}$ Department of Radiology, Universitary Hospital of Puerto Real, Puerto Real, Spain

\section{Email address:}

yosvtit@yahoo.es (I. R. Hernández), acd3202@yahoo.es (A. C. Durán), ana.diez.sspa@juntadeandalucia.es (A. D. Núñez), juan.alija.castro@gmail.com (J. A. Castro),vicente.vega.sspa@juntadeandalucia.es (V. V. Ruiz)

\section{To cite this article:}

Iosvany Rivero Hernández, Antonio Calvo Durán, Ana Diez Núñez, Juan Alija Castro,Vicente Vega Ruiz. Massive Splenic Infarction Secondary to Distal Pancreatectomy for a Blunt Abdominal Trauma. Advances in Surgical Sciences. Vol. 9, No. 1, 2021 , pp. 6-9. doi: $10.11648 /$ j.ass.20210901.12

Received: January 29, 2021; Accepted: February 6, 2021; Published: February 23, 2021

\begin{abstract}
Massive splenic infarction is an uncommon diagnosis that is often overlooked. It is whether asymptomatic or symptomatic with abdominal pain, fever and splenomegaly. There is no consensus on which the best treatment option is, in most cases it is about deciding on conservative or surgical management according to individual patient characteristics. Case presentation: We present the case of a 33-year- old patient with a blunt abdominal trauma while riding a horse, who is taken to the hospital emergency department. A contrast-enhanced abdominal computed tomography is carried out with the diagnosis of a Grade III pancreatic lesion involving the distal portion of the body and tail of the pancreas with associated active bleeding. An urgent laparotomy is performed with a spleen-preserving distal pancreatectomy procedure. During the immediate postoperative phase a massive splenic infarction is diagnosed after a follow-up contrast-enhanced abdominal computed tomography is carried out and successfully treated with conservative measures. Conclusions: It is important to highlight the feasibility of the non-surgical treatment in this kind of spleen lesion, because the spleen preservation proves to be necessary, whenever possible, due to the importance of its immunological role for the high risk of sepsis associated with death and neoplastic processes on those individuals with splenectomy.
\end{abstract}

Keywords: Massive Splenic Infarct, Distal Pancreatectomy, Blunt Abdominal Trauma

\section{Introduction}

Massive splenic infarction is an uncommon diagnosis and is characterized by vessel occlusion, parenchymal ischemia, and subsequent tissue necrosis involving more than half of the spleen. However, finding a patient with abdominal pain and fever is a common situation with a broad range of differential diagnosis among which splenomegaly is included. This could be considered an isolated and common sign in some ailments that manifests with a distended or painful abdomen and could be caused by chronic haemolysis, haematological and nonhaematological neoplastic diseases [1].

The splenomegaly related to a massive splenic infarction is an uncommon finding. It is generally associated with hematologic, oncologic and infectious diseases, thus it is not considered a first- line diagnostic option and is overlooked in over a $25 \%$ of cases [2].

There is very little evidence in the literature regarding this issue, as a result, diagnosis is less likely to be established. It has an acute progression and could be mistaken for an acute abdomen, except that when the diagnosis is made, it could be managed conservatively with serial follow-up imaging tests.

We describe the case of a patient with a massive splenic infarct secondary to a distal pancreatectomy for blunt abdominal trauma with a grade III pancreatic lesion.

\section{Case Presentation}

A 33-year-old woman was brought by emergency medical services to our emergency department with a blunt abdominal and cranial trauma while riding a horse without losing 
consciousness. Hemodynamically stable at the time of arrival with BP 90/60mmHg, Pulse 81, GCS 15, Haemoglobin 13.9 $\mathrm{g} / \mathrm{dl}$, small wound over the left side of the forehead without

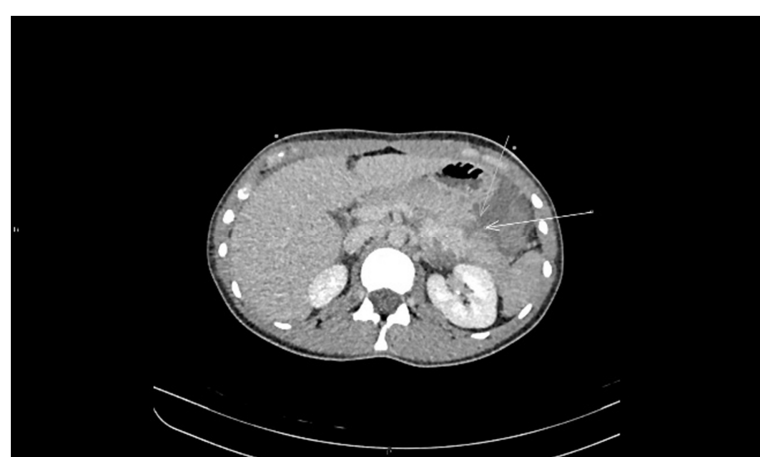

(a)

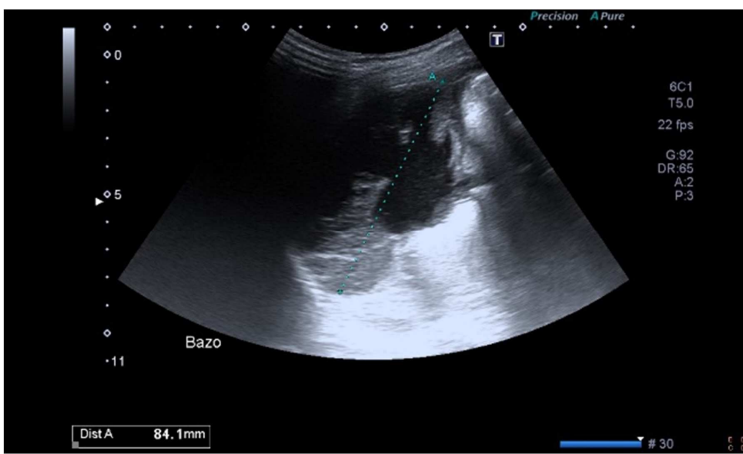

(c) active bleeding and abdominal pain over the left upper quadrant without abdominal tenderness.

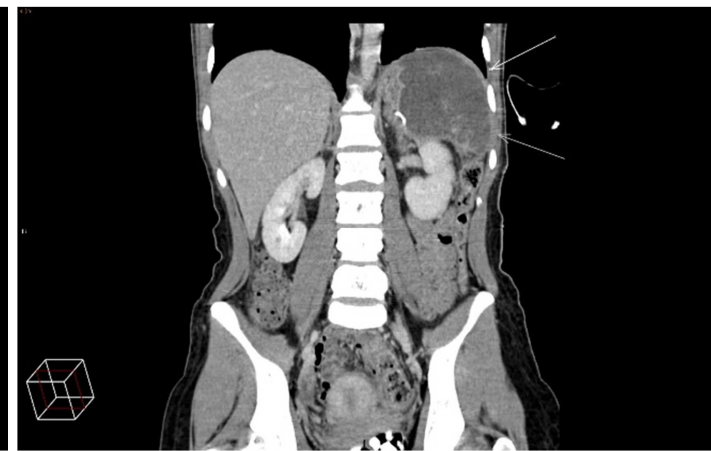

(b)

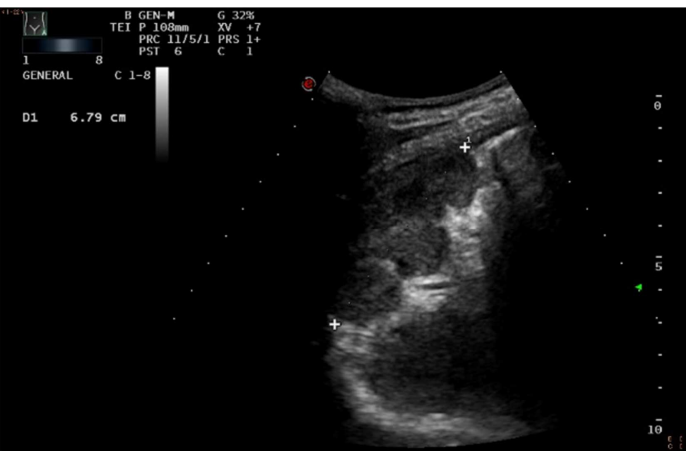

(d)

Figure 1. Diagnosis and evolution of lesions through abdominal sonography and Computed tomography Scan. Complete pancreatic laceration- fracture over the pancreatic body and tail union with associated active bleeding (white arrow) (a); Massive splenic infarction on the 10th post-operative day (white arrow) (b); Follow-up sonography, in the first month after the surgical procedure, of an $8.5 \mathrm{~cm}$ diameter spleen (dash line), and a large hypoechoic area occupying two thirds of the spleen, concerning a necrotic-ischaemic area previously known with preserved areas of normal parenchyma (c); Follow-up sonography over the ninth month of a $7 \mathrm{~cm}$ interpolar diameter spleen (dash line). A one-centimetre small fluid collection/haematoma is identified, having remarkably shrunken concerning previous exams (d).

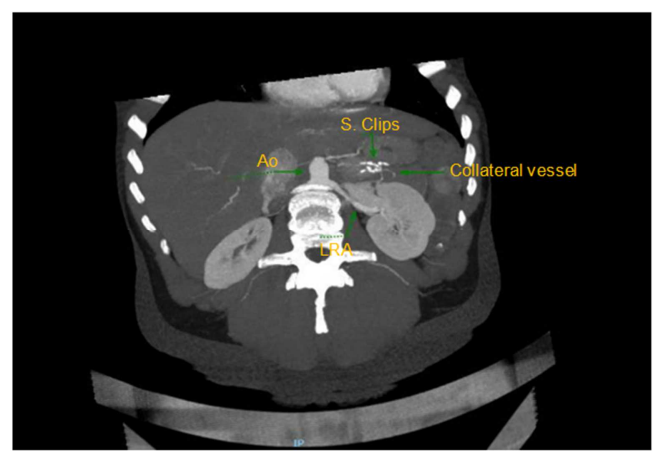

(a)

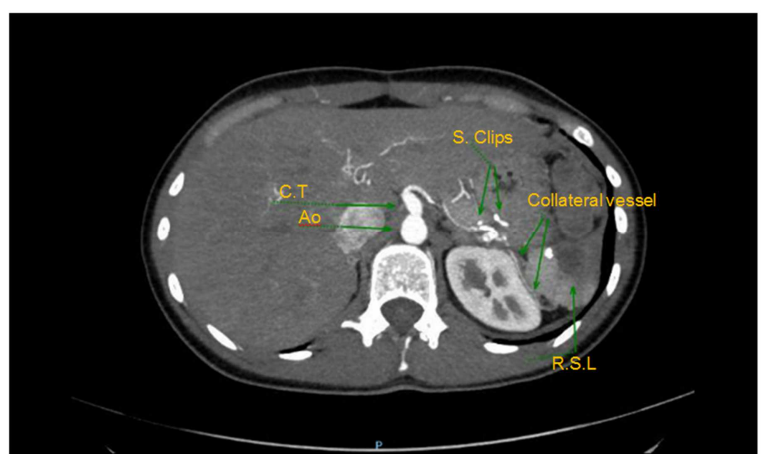

(b)

Figure 2. One year follow-up Contrast-enhanced abdominal computed tomography showing the spleen vascularization. Collateral vessel emerging from the left renal artery, coronal view (a); Collateral vessel supplying the spleen through the superior pole, axial view (b).

A contrast-enhanced abdominal computed tomography is taken and a total laceration of the distal pancreas with a complete transection of the wirsun duct was detected, associated with active bleeding (Figure 1a) and a retroperitoneal peripancreatic hematoma with extension into the intraperitoneal cavity. An urgent spleen and splenic vesselpreserving distal pancreatectomy is performed without incidences. The post-operative period proceeded normally although a gradual increase in platelets count was noticed on a follow-up blood test (Table 1). A contrast-enhanced abdominal computed tomography is taken (Figure 1b) and a massive splenic infarction is detected with an adjacent small associated fluid collection. The treatment consists in percutaneous drainage of the fluid collection with conservative management of the splenic infarction. After a remarkable clinical improvement, the patient was discharged with periodical follow-up, abdominal sonography (Figures 1c, 1d) and blood test on a weekly basis, gradually getting better over time due to 
the favourable recovering of the splenic lesion and function with platelet level normalization (Table 1). For the clinical follow-up, antibiotic prophylaxis is prescribed due to the patient's refusal of vaccination coverage. At one year a followup contrast-enhanced abdominal computed tomography is taken with the following finding: the splenic vessels section and the presence of a collateral vessel emerging from the left renal artery and supplying the spleen through the superior pole (Figure 2a, 2b).

Table 1. Platelet level evolution during the post-operative period.

\begin{tabular}{ll}
\hline Date of test & Platelet count \\
\hline Day 0 & 253000 \\
Post-operative day 6 & 449000 \\
Post-operative day 7 & 596000 \\
Post-operative day 10 & 956000 \\
Post-operative day 12 & 1116000 \\
Post-operative day 14 & 1102000 \\
Post-operative day 27 & 707000 \\
Post-operative day 36 & 467000 \\
Post-operative day 69 & 396000 \\
Post-operative day 289 & 332000 \\
\hline
\end{tabular}

\section{Discussion}

The massive splenic infarction is an uncommon diagnosis and is characterized by vessel occlusion, parenchymal ischemia, and subsequent tissue necrosis involving more than half of the spleen [3]. In most cases, it is associated with a heterogeneous group of entities such as haematological and non-haematological neoplastic diseases, infections, surgeries or traumatisms, and there is no evidence of a predisposing cause around one third of the patients [4]. A short and long-term retrospective study evaluating the splenic vessels patency after a spleen and splenic vessel-preserving distal pancreatectomy reported their compromised patency, mainly over the splenic vein affecting the splenic perfusion, causing splenic infarcts which are generally asymptomatic with a further gradually recovery from the $10^{\text {th }}$ post-operative day $[5,6,15]$.

The onset varies from asymptomatic to an acute abdominal pain over the left upper abdominal quadrant irradiating to the left shoulder, being related or not to splenomegaly or a febrile syndrome, making it indistinguishable from other entities [1, 7]. The laboratory tests are unspecific and leucocytosis, thrombocytosis, or anaemia can be found beside an increase of lactic dehydrogenase in the early stages [2]. The gold standard for the diagnosis is the contrast-enhanced abdominal computed tomography where cuneiforms hypointense areas are seen, corresponding to an infarcted area [8, 9]. In our case, a hypointense homogeneity was detected corresponding to a massive splenic infarct, secondary to the unnoticed splenic vessels section during the distal pancreatectomy. Moreover an associated thrombocytosis was detected with a gradually recovery until the complete normalization of platelet counts over the $10^{\text {th }}$ post-operative week evidencing the splenic function normalization.

The amenable complications secondary to a massive splenic infarct include abscess formation, haemorrhage, or rupture. The splenectomy should be considered for the treatment of any of the aforementioned complications as an urgent measure or should be scheduled as an elective surgery in cases of constant abdominal pain or hypersplenism. There is no consensus about the best treatment for these patients [8, 9], but what is for sure is that they behave as individuals with functional asplenia, what generates a high risk of thrombocytosis and a bigger predisposition to develop sepsis by encapsulated bacteria such as Streptococcus pneumoniae, Haemophilus influenzae tipe $\mathrm{b}$ and Neisseria meningitidis [10], hence vaccination against these is mandatory. Furthermore, the platelet count follow-up, as well as the follow-up of the splenic size through a periodical abdominal sonography, are the cornerstone of the medical treatment $[3$, 9]. In our case, we did not experience any complication associated with the medical management and the spleen recovered approximately the $77 \%$ of its original dimension before the patient's accident, developing a supplying vascularization from the left renal artery [11].

\section{Conclusion}

Our particular interest in this case lies in the feasibility of the medical treatment that has allowed to keep the splenic function due to the importance of its immunological role for the high risk of sepsis associated with death and neoplastic processes on those individuals with splenectomy.

Therefore, the spleen preservation proves to be necessary whenever possible, in the splenic trauma and pancreatic affections which require a distal pancreatectomy, or in the traumatic pancreatic lesions, with spleen preservation and conservation or division of the splenic vessels (Warshaw procedure). These surgical procedures have shown its feasibility $[6,12-15]$.

\section{Abbreviations}

Ao: Aorta; C. T: Celiac Trunk; L. R. A: Left renal artery; R. S. L: Residual splenic lesion; S. Clips: Surgical clips

\section{Ethics Approval and Consent to Participate}

Not applicable.

\section{Consent for Publication}

Informed written consent was obtained from the patient for the publication of the clinical details and clinical images.

\section{Authors' Contributions}

Iosvany $\mathrm{R}$. $\mathrm{H}$ was the major contributor in writing and editing the manuscript. Antonio C. D made substantial contributions to the conception and design of this case report. Ana D. $\mathrm{N}$ and Vicente V. R were involved in the patient's 
treatment. Juan A. C contributed with the patient radiological follow-up and the images selection. All authors read and approved the final manuscript.

\section{Funding}

None.

\section{Availability of Data and Materials}

All relevant data are provided in the manuscript.

\section{Competing Interests}

The authors declare that they have no competing interests.

\section{Acknowledgements}

Not applicable.

\section{References}

[1] Friedman-Klabanoff D, Ball A, Rutare S, McCall N, et al. Three Rwandan Children with Massive Splenomegaly and Epstein-Barr Virus-associated Lymphoproliferative Disorders: Case Presentations and the Literature Review. $J$ Pediatr Hematol Oncol. 2016; 38 (5): e158-61.

[2] Kranidiotis G, Efstratiadis E, Kapsalakis G, Loizos G, et al. Splenic infarcts as a rare manifestation of parvovirus B19 infection. IDCases. 2016; 4: 62-4.

[3] Bokman Christine L.; Sfeir Maroun; Chahwala Veer; Ginzburg Enrique. Spontaneous massive splenic infarction in the setting of renal transplant and septic shock: a case report and review of the literature. Case Rep Med. 2014.

[4] Arenal Vera, J. J. et al. Splenic infarction secondary to acute pancreatitis. Rev. Esp. Enferm. Dig. 2008; 100 (5): 300-303.
[5] Y.-S. Yoon, K. H. Lee, H.-S. Han, J. Y. Cho and K. S. Ahn. Patency of splenic vessels after laparoscopic spleen and splenic vessel-preserving distal pancreatectomy. British Journal of Surgery 2009; 96: 633-640.

[6] Sun et al. Clinical efficacy of spleen-preserving distal pancreatectomy with or without splenic vessel preservation. A Meta-analysis. Medicine (2017) 96: 48.

[7] Antopolsky M, Hiller N, Salameh S, Goldshtein B, et al. Splenic infarction: 10 years of experience. Am J Emerg Med. 2009; 27 (3): 262-5.

[8] F. Fatjó, J. Ramos, A. Culla y J. M. Grau. Splenic infarctions: an uncommon cause of febril syndrome. Med Clin (Barc) 2002; 119 (9): 356-9.

[9] D'Angelo G, Marseglia L, Russo T, Gitto E, et al. Long term follow up of a massive, conservatively treated, splenic infarction in a young adolescent. Pediatr Int. 2017; 59 (11): 1210-2.

[10] Lamsfus-Prieto JA et al. Prevention of sepsis in asplenic patients. Cir Esp. 2007; 81 (5): 247-51.

[11] Skandalakis JE, Colborn GL, Pemberton LB, Skandalakis TN, Skandalakis LJ, Gray SW. The surgical anatomy of the spleen. Prob Gen Surg 7 (1): 1-17, 1990.

[12] Carrére et al.: Spleen-preserving distal pancreatectomy with excision of splenic artery and vein: A case matched comparison with conventional distal pancreatectomy with splenectomy. World J Surg (2007) 31: 375-382.

[13] A. L. Warshaw, "Conservation of the spleen with distal pancreatectomy," JAMA Surgery, vol. 123, no. 5, pp. 550-553, 1988.

[14] Ferrone et al, "Twenty-Three Years of the Warshaw Operation for Distal Pancreatectomy With Preservation of the Spleen”. Ann Surg 2011; 253: 1136-1139.

[15] Kimura et al, "A case of complete splenic infarction after laparoscopic spleen-preserving distal pancreatectomy. BMC Surgery (2018) 18: 22. 